\title{
Prognostic Value of Tumour-Infiltrating Lymphocytes Changes between Pre and Post-Neoadjuvant Treatment and in Residual Disease in Triple Negative Breast Cancer
}

Herrero-Vicent $C^{1^{*}}$, Calatrava $A^{2}$, Ramírez $M^{3}$, Gavilá $J^{4}$, Guerrero $A^{4}$, Hernández-Serra $A^{3}$, LopezGuerrero $J A^{3}$, Guillem-Porta $V^{4}$ and Ruiz-Simón $A^{4}$

${ }^{1}$ Medical Oncology Department, Hospital Provincial de Castellón, Spain

${ }^{2}$ Pathology Department, Valencian Institute of Oncology, Spain

${ }^{3}$ Molecular Biology Department, Valencian Institute of Oncology, Spain

${ }^{4}$ Medical Oncology Department, Valencian Institute of Oncology, Spain

\begin{abstract}
Introduction: Triple-negative breast cancer (TNBC) is a heterogeneous disease with different biological behaviours and aggressiveness. Cancer immune edition has been revealed as a major hallmark in cancer. In patients treated with neoadjuvant chemotherapy (NAC) it is the perfect setting to study possible changes in tumour-infiltrating lymphocyte (TIL) levels since we have histological sample pre-treatment after NAC lacking pathological complete response (pCR). Several recent clinical studies have evaluated TILs in TNBC patients with different methodological approaches. Recently, the International TILs Working Group proposed that full sections are preferred over core biopsies.

Material and methods: Using our prospective maintained BC database we identified 164 TNBC patients treated with NAC between 1998 and 2015 with enough samples of diagnostic biopsy and after surgery. Evaluation of TILs after NAC was following a standardized methodology for visual assessment on haematoxylin-eosin (HE) full sections and stromal TIL was measured as a continuous variable. The primary end point was prognostic value of TILs in residual disease. The secondary endpoint was influence of change in TIL score before and after NAC in disease free survival (DFS). DFS was analysed with Kaplan-Meier method and groups were compared with long-rank test. Univariate and multivariate Cox models were used to generate hazard ratios (HR) for determining associations between variables and DFS.
\end{abstract}

Results: 103 TNBC patients had residual disease after NAC. At a median follow-up of 78 months, lymphocytepredominant breast cancer (LPBC) was associated with better DFS; the 6-year Kaplan-Meier estimates for DFS were $66.2 \%$ and $23 \%$ for patients with LPBC and non-LPBC, respectively $(p=0.001)$. About influence of change in TIL score before and after NAC in disease free survival (DFS); median DFS in non-changes-group was 40 months $(95 \% \mathrm{Cl}$ 8.1-71 months), decrease TILs group 154 months (95\% Cl 9-371 months), and increase TILs group was not reached $(p=0.001)$. By univariate analysis, increase TILs levels between and after NAC vs. decrease or non-changes levels HR 4.01, (95\% Cl 1.69-9.59; $p=0.002)$. And by multivariate analysis TILs levels in residual disease $\mathrm{HR} 2.92(95 \% \mathrm{Cl}$ $1.05-9.66 ; p=0.040)$

Conclusions: TILs changes between pre and post-neoadjuvant treatment and in residual disease in triple negative breast cancer proportionate relevant prognostic in this study TNBC.

\section{Keywords}

Triple-negative breast cancer, Tumour-infiltrating lymphocytes, Residual disease, Prognostic value

\section{Abbreviations}

TNBC: Triple-Negative Breast Cancer; Pcr: Pathologic Complete Response; TIL: Tumour-Infiltrating Lymphocytes; HE: Haematoxylin-Eosin; LPBC: Lymphocyte-Predominant Breast Cancer; HR: Hazard Ratios; COR: Receiver Operating Characteristic; NAC: Neoadjuvant Chemotherapy; RT: Adjuvant Radiotherapy; LVI: Lymph Vascular-Invasion; DCIS: Ductal Carcinoma in Situ; OS: Overall Survival; Treg: Regulatory T Cells; TAM: Tumour Associated Macrophages; MDSC: MyeloidDerived Suppressor Cells 
Citation: Herrero-Vicent C, Calatrava A, Ramírez M, et al. (2019) Prognostic Value of Tumour-Infiltrating Lymphocytes Changes between Pre and Post-Neoadjuvant Treatment and in Residual Disease in Triple Negative Breast Cancer. Annals Gynecol Obstet 3(1):50-58

\section{Introduction}

Triple-negative breast cancer (TNBC) is a heterogeneous disease with different biological behaviours and aggressiveness. Cancer immune edition has been revealed as a major hallmark in cancer. As tumour evolve, their specific stroma turns into a microenvironment with overwhelming density of immunosuppressive cells such as regulatory T cells (Treg), tumour associated macrophages (TAM), myeloid-derived suppressor cells (MDSC) and T-cell of Th-2 type response among others [1]. Normal breast tissue does not contain large aggregates of immune cells, but breast tumours and their adjacent stroma display higher levels of immune-cells infiltrates [2]. In breast cancer, T infiltrating lymphocytes (TILs) as a whole (helper T cells, cytotoxic T cells, Treg) nowadays represent a putative prognostic and predictive biomarker $[3,4]$.

Recently, the International TILs Working Group proposed a standardized methodology to measure TILs in breast cancer though visual assessment on haematoxylin and eosin sections [5]. Main conclusions of this consensus were that full sections are preferred over biopsies, and TILs should be measured as a continuous variable as a percentage. Besides, stromal compartment is at the moment the location where TILs may proportionate more value information from clinical point of view [6].

In patients treated with neoadjuvant chemotherapy (NAC) it is the perfect setting to study possible changes in TIL levels since we have histological sample pre-treatment and after NAC lacking pathological complete response (pCR) [7]. However, NAC selects a subpopulation of chemotherapy-resistant tumours cells (non-pCR $55-70 \%$ in TNBC), and patients lacking $\mathrm{PCR}$ have unfavourable recurrence-free survival and overall survival [8].

Basal TILs or pre-NAC have a predictive value for response to chemotherapy in TNBC $[4,6]$. However, clinic-pathological biomarkers are not sufficiently accurate to distinguish the patients who could relapse. In patients without PCR, TILs in residual carcinoma after NAC may therefore help in assessing risk and stratifying adjuvant treatment in patients and new strategies should be considered to improve the outcome of TNBC patients [9].

We aimed to investigate the prognostic value of TIL changes before and after neoadjuvant treatment and to confirm prognostic value of TILs in residual disease.

\section{Materials and Methods}

\section{Patients}

A retrospective, single-institution study was conducted at Valencian Institute of Oncology (Valencia, Spain) with ethics committee approval. We based on a maintained database for patients with locally advanced breast cancer treated with neoadjuvant treatment between 1998 and 2015.

Patients were included in the study if they had TN breast cancer (ER- PR- and HER2-). The threshold for ER and PR positivity were set at $1 \%$ using immunochemical staining. Patients were treated with neoadjuvant chemotherapy (NAC) based on anthracycline and taxane regimen. Clinic-pathological information was obtained from the database; age, grade, tumour size, histological classification, lymph node status, lymph vascular-invasion, ki67 and adjuvant treatment.

\section{Samples}

Haematoxylin-eosin (HE) samples were reviewed by a breast pathologist (FG) who was blinded to the patient profiles. The evaluation of TILs was following a standardized methodology for visual assessment on HE sections and TILs were measured as a continuous variable [4]. He defined TIL score as the proportion of the stromal area infiltrated by lymphocytes following the recommendations of the International TIL breast cancer working group. Recently, the International TILs Working Group proposed that full sections are preferred over core biopsies [5]. Representative slide containing relatively high amount of lymphocytic infiltration around invasive cancer was selected for each patient. Due to the heterogeneity of TILs, with different intensities of lymphocytes in different areas, hot spots at the invasive edge were avoided.

\section{Outcomes}

Pathological complete response ( $p C R$ ) was defined as no residual invasive carcinoma in breast (ypTO or ypTis) and negative lymph node status (ypNO).

Prognostic information was retrospectively obtained from a maintained clinical database. Disease free survival (DFS) was defined as the period of time between surgery and breast cancer relapsed, death of any cause or latest follow-up.

\section{Statistical analysis}

Data were analysed using SPSS version 22.0. We categorized lymphocyte-predominant breast cancer (LPBC) cutoff according to a COR analysis. The association between clinical and pathologic parameters was tested with $\mathrm{X}^{2}$ test for categorical variables. Mean differences were studied with the T-test. DFS was analysed with Kaplan-Meier method and groups were compared with long-rank test. For univariate analysis, the difference between survival function was calculated using univariate Cox model. Multivariate

*Corresponding author: Herrero-Vicent C, MD, PHD, Medical Oncology Department, Hospital Provincial de Castellón, Av. del Dr. Clarà, 19, 12002 Castelló de la Plana, Castelló, Spain, Tel: $+34-620519816$

Accepted: October 22, 2019

Published online: October 24, 2019

Citation: Herrero-Vicent C, Calatrava A, Ramírez M, et al. (2019) Prognostic Value of Tumour-Infiltrating Lymphocytes Changes between Pre and Post-Neoadjuvant Treatment and in Residual Disease in Triple Negative Breast Cancer. Annals Gynecol Obstet 3(1):50-58 


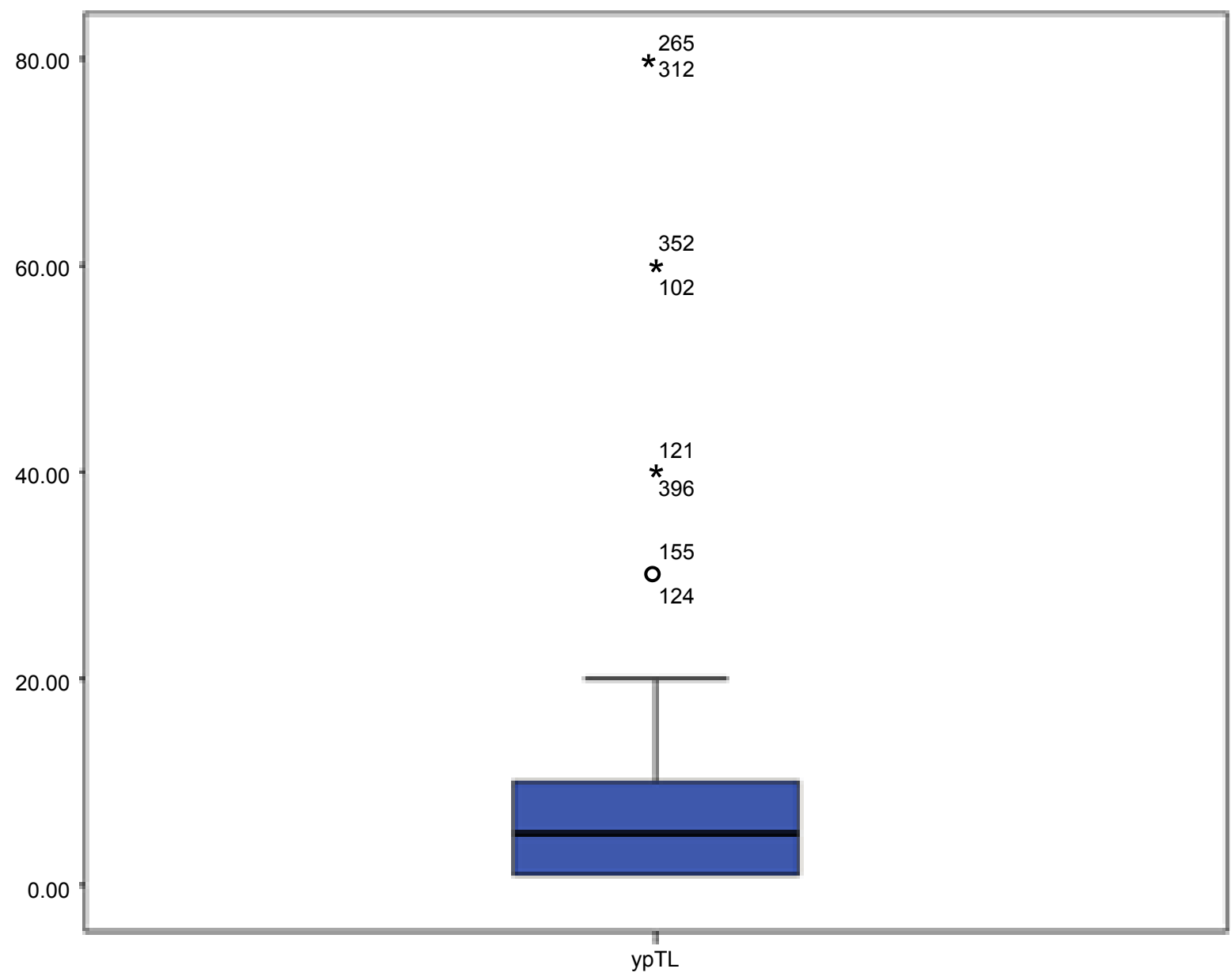

Figure 1: Boxplot of TILs levels in residual disease.

Cox proportional hazard regression model was used for multivariate analysis, which included all prognostic factors that were significant in univariate analysis.

\section{Results}

\section{Patients' characteristics and TILs in residual dis- ease}

A total of 756 patients diagnosed with locally advanced invasive carcinomas and treated with NAC followed by surgery were identified from 1998 to 2015 . Based on the criteria described, 181 patients were diagnosed with TNB. Of them, 164 patients (90.6\%) had enough sample available before and after NAC. In our study, 61 patients (37.2\%) presented pCR (ypTO/ypTis ypNO) and 103 patients (62.8\%) presented residual disease.

The detailed distribution of TILs in residual disease is detailed in Figure 1. Median TIL in residual disease was 5\% (0$80 \%$ ) Main samples (86.7\%) presented TILs from $1 \%$ to $10 \%$. Lymphocyte-predominant breast cancer (LPBC) cut-off was > $1 \%$ lymphocytic infiltration tumour stroma $(p=0.001)$. We categorized LPBC 60 patients (58.3\%) meanwhile 43 patients (44.7\%) were non-LPBC.

Detailed distributions of clinical and pathological charac-
Table 1: Detailed distributions of clinical and pathological characteristics between LPBC group and non-LPBC group.

\begin{tabular}{|l|l|l|l|}
\hline Variable & \multicolumn{2}{|l|}{ TIL in residual disease } & $X^{2}$ \\
\hline & High & Low & \\
\hline Age < 40 y & $30(49 \%)$ & $27(64 \%)$ & p.094 \\
\hline Clinical stage cTNM & & & \\
\hline Ila & $21(34.4 \%)$ & $5(11.9 \%)$ & $p .002$ \\
\hline Ilb & $23(37.7 \%)$ & $9(21.4 \%)$ & \\
\hline IIla & $10(16.4 \%)$ & $10(23.8 \%)$ & \\
\hline IIlb & $1(1.6 \%)$ & $9(21.4 \%)$ & \\
\hline IIlc & $6(9.8 \%)$ & $9(21.4 \%)$ & \\
\hline Histology & & & \\
\hline Ductal & $39(63.9 \%)$ & $25(60 \%)$ & $p .473$ \\
\hline Lobulillar & $22(36.1 \%)$ & $17(40 \%)$ & \\
\hline No DCIS in core biopsy & $40(65.6 \%)$ & $31(73.8 \%)$ & $p .363$ \\
\hline Grade 3 & $16(26 \%)$ & $11(26 \%)$ & $p .675$ \\
\hline Nolymphovascular invasion & $45(73.8 \%)$ & $35(83.3 \%)$ & $p .131$ \\
\hline Ki67 < 50\% & $8(16 \%)$ & $4(10 \%)$ & p.270 \\
\hline High TIL in core biopsy & $54(88.5 \%)$ & $42(100 \%)$ & p.022 \\
\hline NAC & & & \\
\hline & & & \\
\hline
\end{tabular}


Citation: Herrero-Vicent C, Calatrava A, Ramírez M, et al. (2019) Prognostic Value of Tumour-Infiltrating Lymphocytes Changes between Pre and Post-Neoadjuvant Treatment and in Residual Disease in Triple Negative Breast Cancer. Annals Gynecol Obstet 3(1):50-58

\begin{tabular}{|l|l|l|l|}
\hline Antracyclines + taxanes & $55(90 \%)$ & $40(95 \%)$ & p.254 \\
\hline Residual disease stagey pTNM & & & \\
\hline I & $31(50.8 \%)$ & $6(14.3 \%)$ & p.002 \\
\hline Ila & $16(26.2 \%)$ & $9(21.4 \%)$ & \\
\hline Illb & $6(9.8 \%)$ & $6(14.3 \%)$ & \\
\hline IIla & $6(9.8 \%)$ & $15(35.7 \%)$ & \\
\hline IIlb & $1(1.6 \%)$ & $0(0 \%)$ & \\
\hline IIlC & $1(1.6 \%)$ & $6(14.3 \%)$ & \\
\hline Residual tumor & & & \\
\hline Multifocal & $13(21.3 \%)$ & $10(23.8 \%)$ & $p .649$ \\
\hline DCIS in residual disease & $9(14.8 \%)$ & $9(21.8 \%)$ & $p .311$ \\
\hline No LVI in residual disease & $12(19.7 \%)$ & $16(38.1 \%)$ & $p .048$ \\
\hline Breast conservative surgery (BCS) & $34(55.7 \%)$ & $9(22 \%)$ & p.001 \\
\hline Adjuvant RT & $52(85.2 \%)$ & $40(95.2 \%)$ & p.252 \\
\hline
\end{tabular}

teristics between LPBC group and non-LPBC group are summarized in Table 1. There were no differences between median ages, histology, DCIS associated, histological grade, lymph vascular -invasion, ki67 marker, neither NAC regimen. However, LPBC group presented higher clinical stage II, $(72.1 \%$ vs. $33.3 \% p=002)$ and breast conservative surgery $(55.7 \%$ vs. $22 \% p=0.001$ ) than non-LPBC. In residual disease, there were no differences between residual focus neither DCIS associated. However, non-LPBC presented higher pathological stage (stage III $50 \%$ vs. $13.2 \%, p=0.002$ ) and lymph vascular -invasion $(p=0.048)$.

\section{TILs changes between pre and post NAC}

103 with non-pCR, pair-matched (pre and post NAC) were available and TIL in core biopsy and surgical samples were compared. Changes in TIL scores were classified as decrease, increase and no-change. The proportions cases in each subgroup are summarized: 50 patients $(48.6 \%)$ had no-change, 26 patients $(25.2 \%)$ decrease TILs score after NAC and 27 patients (26.2\%) increase TIL score. The detailed distribution of TILs before and after NAC is detailed in Figure 2.

The detailed distributions of clinical and pathological characteristics and treatment are summarized in Table 2. There were no differences between median age, histology, DCIS associated, histological grade, lymph vascular-invasion, ki67 marker, neither NAC regimen. However, no changes TILs group presented higher clinical stage III, (62\% vs. $30.7 \%$ and $22.2 \%, p=0.014$ ) than decreased and increased TILs groups, respectively. In residual disease, there were no differences between residual focus neither DCIS associated, lymph vascular-invasion neither pathological stage.

\section{Prognostic value of TIL in residual disease and TIL score changes between pre and post NAC treatment}

At a median follow-up of 78 months, 6 year-recurrence was $66.2 \%$ in non-LPBC group and $23 \%$ in LPBC ( $p=0.001)$. Figure 3 illustrates disease-free survival curves according to
Table 2: Detailed distributions of clinical and pathological characteristics between non-changes level group, decrease and increased TILs level groups.

\begin{tabular}{|c|c|c|c|c|}
\hline \multirow[t]{2}{*}{ Variable } & \multicolumn{3}{|c|}{$\begin{array}{l}\text { TILs changes between pre and post } \\
\text { NAC }\end{array}$} & \multirow[t]{2}{*}{$x^{2}$} \\
\hline & Increase & Decreased & No-changes & \\
\hline Age $<40 y$ & $14(52 \%)$ & $15(57 \%)$ & $28(56 \%)$ & p.905 \\
\hline \multicolumn{5}{|l|}{ cTNM } \\
\hline Ila & $9(33.3 \%)$ & $8(30.8 \%)$ & $9(18 \%)$ & p.014 \\
\hline $\mathrm{Ilb}$ & $12(44.4 \%)$ & $10(38.5 \%)$ & $10(20 \%)$ & \\
\hline IIla & $5(18.5 \%)$ & $5(19.2 \%)$ & $10(20 \%)$ & \\
\hline IIIb & $0(0 \%)$ & $1(3.8 \%)$ & $9(18 \%)$ & \\
\hline IIIC & $1(3.7 \%)$ & $2(7.7 \%)$ & $12(24 \%)$ & \\
\hline \multicolumn{5}{|l|}{ Histology } \\
\hline Ductal & 17 (63\%) & $13(50 \%)$ & $29(58 \%)$ & p.098 \\
\hline Lobulillar & $10(37 \%)$ & $13(50 \%)$ & $33(42 \%)$ & \\
\hline No DCIS in biopsy & $16(59.3 \%)$ & $18(69.2 \%)$ & $37(74 \%)$ & p.795 \\
\hline Grade 3 & $9(33.3 \%)$ & $6(23.1 \%)$ & $12(24 \%)$ & p.451 \\
\hline No LVI in biopsy & $19(70.4 \%)$ & $22(84.6 \%)$ & $32(78 \%)$ & p.050 \\
\hline Ki67 < 50\% & $2(7.4 \%)$ & $4(16 \%)$ & $8(16.8 \%)$ & p.610 \\
\hline Low TIL pre NAC & $28(100 \%)$ & 20 (76.9\%) & $49(98 \%)$ & p.014 \\
\hline $\begin{array}{l}\text { Antracyclines } \\
+ \text { taxanes }\end{array}$ & 26 (96\%\%) & 23 (89\%) & $46(92 \%)$ & p.565 \\
\hline \multicolumn{5}{|l|}{ урTNM } \\
\hline I & $11(40.7 \%)$ & $11(42.3 \%)$ & 15 (30\%) & p.384 \\
\hline Ila & $5(18.5 \%)$ & $10(38.5 \%)$ & $10(20 \%)$ & \\
\hline $\mathrm{Ilb}$ & $5(18.5 \%)$ & $1(3.8 \%)$ & $6(12 \%)$ & \\
\hline IIla & $5(18.5 \%)$ & $3(11.5 \%)$ & $13(26 \%)$ & \\
\hline IIIb & $0(0 \%)$ & $0(0 \%)$ & $1(2 \%)$ & \\
\hline IIIC & $1(3.7 \%)$ & $1(3.8 \%)$ & $5(10 \%)$ & \\
\hline \multicolumn{5}{|l|}{ Residual tumor } \\
\hline Multifocal & $5(18.5 \%)$ & 7 (26.9\%) & $11(22 \%)$ & p.393 \\
\hline DCIS specimen & $4(14.8 \%)$ & $5(19.2 \%)$ & $9(18 \%)$ & p.864 \\
\hline Low TIL after NAC & $0(0 \%)$ & $6(23.1 \%)$ & $36(72 \%)$ & p.001 \\
\hline LVI specimen & $6(22.2 \%)$ & $6(23.1 \%)$ & $16(32 \%)$ & p.671 \\
\hline BCS & $15(55.6 \%)$ & $13(50 \%)$ & $15(30 \%)$ & p.050 \\
\hline Adjuvant RT & $23(85.2 \%)$ & $21(80.8 \%)$ & $48(96 \%)$ & p.112 \\
\hline
\end{tabular}

LPBC and non-LPBC groups post chemotherapy.

By univariate and multivariate analysis, variables associated with an increased recurrence are described in Tables 3 and Table 4, respectively.

By univariate analysis, TILs levels in residual disease after NAC HR 4.27, (95\% Cl 2.29-7.98; $p=0.001)$. However, by multivariate analysis TILs levels in residual disease HR 1.59 (95\% Cl 0.70-3.59; $\mathrm{p}=0.265$ ).

On the other hand, TILs score changes between pre and post NAC treatment prognostic value was also evaluated. 6 
Citation: Herrero-Vicent C, Calatrava A, Ramírez M, et al. (2019) Prognostic Value of Tumour-Infiltrating Lymphocytes Changes between Pre and Post-Neoadjuvant Treatment and in Residual Disease in Triple Negative Breast Cancer. Annals Gynecol Obstet 3(1):50-58



Figure 2: TILs changes before and after NAC.

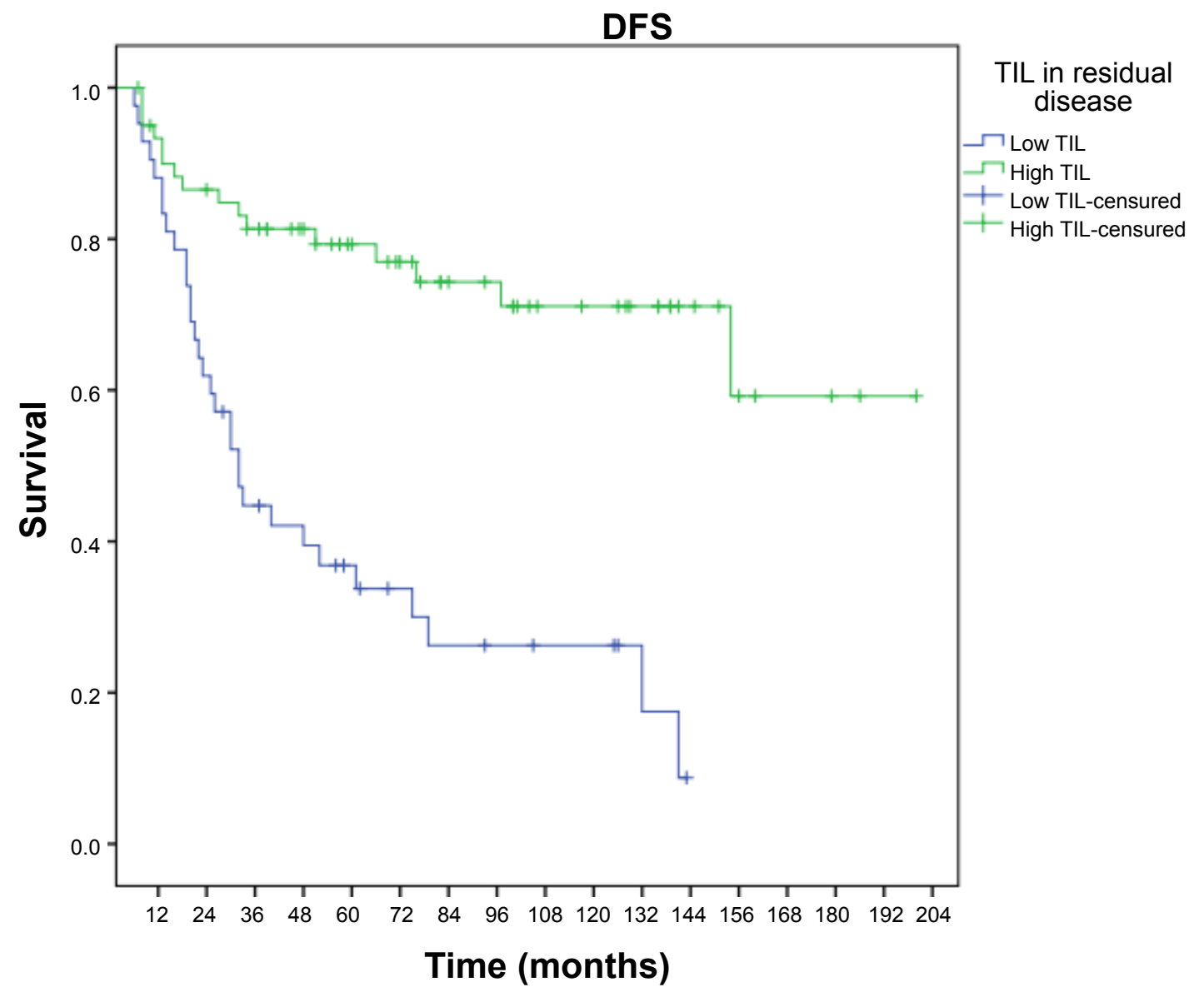

Figure 3: Disease-free survival curves according to LPBC and non-LPBC groups (post-chemotherapy). 
Citation: Herrero-Vicent C, Calatrava A, Ramírez M, et al. (2019) Prognostic Value of Tumour-Infiltrating Lymphocytes Changes between Pre and Post-Neoadjuvant Treatment and in Residual Disease in Triple Negative Breast Cancer. Annals Gynecol Obstet 3(1):50-58

Table 3: Univariate analysis details: Variables associated with an increased recurrence.

\begin{tabular}{|c|c|c|}
\hline Variable & HR (Cl 95\%) & p \\
\hline Clinical stage TNM III vs. II & HR 2.22 (Cl 1.51-4.21) & $p=0.001$ \\
\hline cT $;>2 \mathrm{~cm}$ vs. $<2 \mathrm{~cm}$ & HR $1.76(\mathrm{Cl} 0.24-12.77)$ & $p=0.567$ \\
\hline $\mathrm{cN} ; \mathrm{cN}+$ vs. cNO & HR 2.50 (CI 1.39-4.49) & $p=0.020$ \\
\hline Grade III vs. I-II in core biopsy & HR 1.39 (Cl 0.79-2.44) & $p=0.243$ \\
\hline Low TIL vs. High TIL in core biopsy & HR 3.44 (Cl 1.78-6.65) & $p=0.001$ \\
\hline TIL changes pre and post NAC; Decrease or non-changes vs. increase & HR 4.01 (Cl 1.69-9.59) & $p=0.002$ \\
\hline Pathologic complete response; Non-pCR vs. pCR & HR 2.89 (Cl 1.56-5.36) & $p=0.001$ \\
\hline Residual disease stage ypTNM II-III vs. I & HR 4.28 (Cl 1.91-9.59) & $p=0.001$ \\
\hline ypT; residual breast tumor $>2 \mathrm{~cm}$ vs. $<2 \mathrm{~cm}$ & HR 3.01 (Cl 1.66-5.44) & $p=0.002$ \\
\hline ypN; ypN + vs. ypNO & HR 3.66 (Cl 2.19-6.12) & $p=0.001$ \\
\hline Low TIL vs. High TIL in residual disease & HR 4.27 (Cl 2.29-7.98) & $p=0.001$ \\
\hline
\end{tabular}

Table 4: Multivariate analysis details: Variables associated with an increased recurrence.

\begin{tabular}{|l|l|l|}
\hline Variable & HR (Cl 95\%) & p \\
\hline CN; N + vs. NO & HR 2.82 (Cl 1.21-6.80) & $p=0.017$ \\
\hline TIL changes pre and post NAC; Decrease or non-changes vs. increase & HR 3.10 (Cl 1.05-9.66) & $p=0.040$ \\
\hline ypT; tumor residual > 2 cm vs. <2 cm & HR 2.92 (Cl 1.51-5.25) & $p=0.001$ \\
\hline Low TIL vs. High TIL in residual disease & HR 1.59 (Cl 0.70-3.59) & $p=0.263$ \\
\hline Low TIL vs. High TIL in core biopsy & HR 1.51 (Cl 0.31-7.29) & $p=0.604$ \\
\hline
\end{tabular}

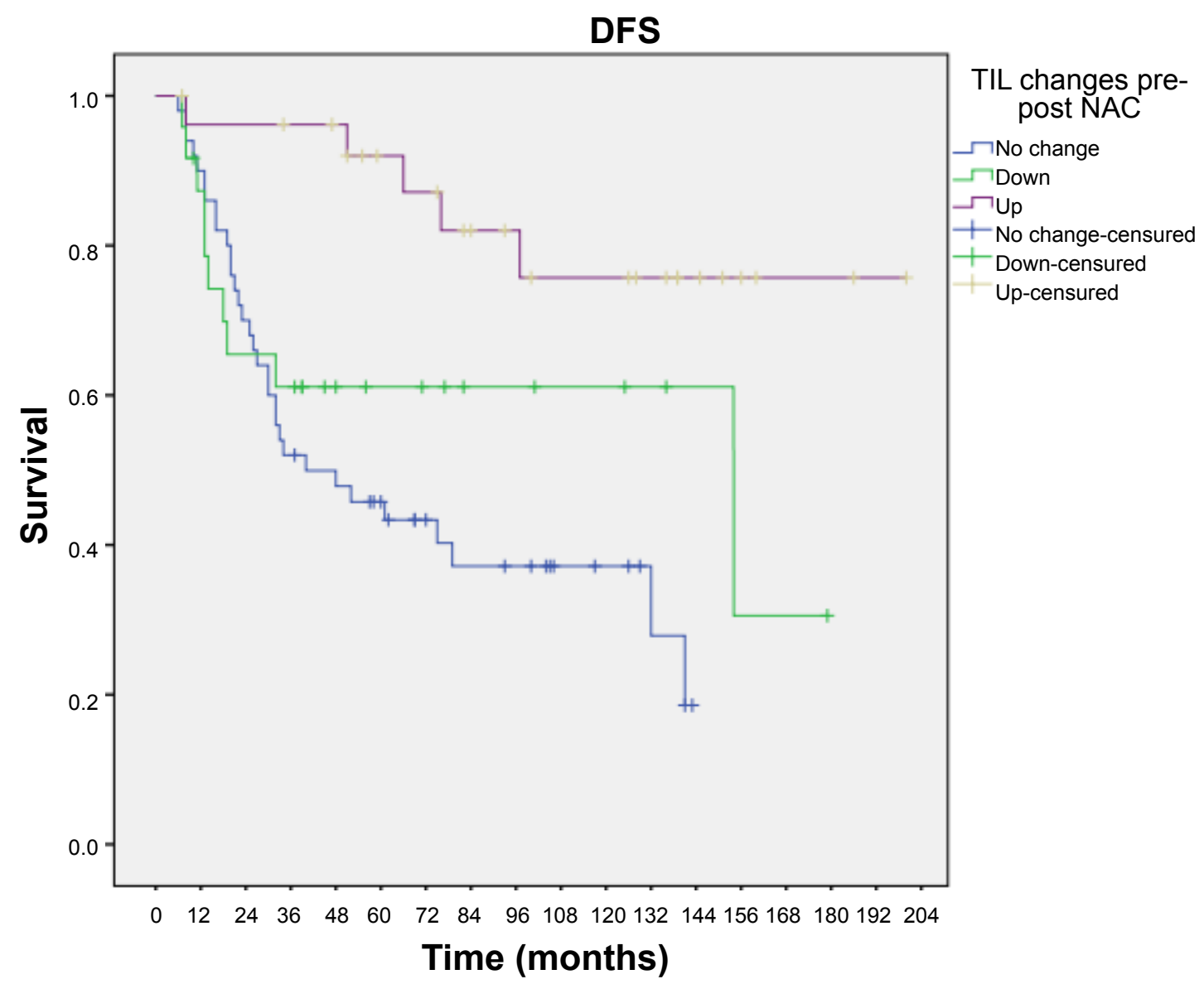

Figure 4: Disease-free survival curves according to TILs level changes groups. 
Citation: Herrero-Vicent C, Calatrava A, Ramírez M, et al. (2019) Prognostic Value of Tumour-Infiltrating Lymphocytes Changes between Pre and Post-Neoadjuvant Treatment and in Residual Disease in Triple Negative Breast Cancer. Annals Gynecol Obstet 3(1):50-58

year-recurrence was $56.6 \%$ in non-changes group, $38.9 \%$ in decrease TIL score group and $16.5 \%$ in increase TIL score group $(p=0.001)$.

TILs score changes proved to have significant prognostic value ( $p=0.001$ ) regarding DFS. Median DFS in non-changesgroup was 40 months (95\% Cl 8.1-71 months), decreased TILs group 154 months (95\% Cl 9-371 months), and increased TILs group was not reached $(p=0.001)$.

Figure 4 illustrates disease-free survival curves according to no changes/decrease and increased TIL score groups.

By univariate analysis, increase TILs score between and after NAC vs. decrease or non-changes levels HR $4.01,(95 \% \mathrm{Cl}$ 1.69-9.59; $p=0.002$ ). And by multivariate analysis TILs levels in residual disease HR $2.92(95 \% \mathrm{Cl} 1.05-9.66 ; \mathrm{p}=0.040)$.

\section{Discussion}

In summary, we investigate the utility of TILs following the scoring system of the International TIL Breast Cancer Working Group [5]. In this retrospective study, we report the prognostic value of TILs in residual disease after NAC.

On the other hand, data support the immunomodulatory effects of chemotherapy [10]. Indeed, chemotherapy might relieve immunosuppression and reset attenuated functional immunity. An immune response could also be triggered by chemotherapy-induced cell death; this response might contribute to eliminating the remaining viable tumour in the breast and distant micro metastasis [8]. In this study, we report the prognostic value of TIL changes between core biopsy and surgical samples in TNBC.

\section{Cut-off point}

We recognised heterogeneity of TILs cut-off. TILs were analysed as a continuous variable and as a dichotomous ortrichotomous variable with one or two cut-off [9,11-13] there is no formal recommendation for clinical relevant threshold yet.

The German Breast Cancer Group (GBC) determined high TILs-LPBC (60-100\%), intermediate TILs (11-59\%) and low TIL $(0-10 \%)[11]$. However, GBC take into account stromal and intratumoral TILs. Although, Hida, et al. divided also into three different groups but take into account just stromal TILs $[1,14]$.

On the other hand, The TIL Working Group describes the term lymphocytic-predominant breast cancer (LPBC) for tumours that harbour a very high immune infiltrate $>50 \%$ [4]. And theses LPBC may represent from $13-30 \%$ of breast cancer overall and there are differences depending on the specific subtypes, being more frequent in TNBC [11].

In one of the largest studied cohorts of women with lymph-node-positive disease, the median percentage of stromal tissue infiltrated with TILs was $20 \%$ in TNBC [9]. In our study median TIL in residual disease was $5 \%(0-80 \%)$. Main samples (86.7\%) presented TILs from $1 \%$ to $10 \%$. We performed a ROC curve and we categorized LPBC as the involving $>1 \%$ stromal TILs. Our study had several limitations in terms of analytical validation; we did not prove the ro- bustness and reproducibility of the TILs cut-off. However, the richness and diversity of antitumor immune response is, of course, severely underestimated by assessment with HE [15-17].

\section{Prognostic value of TILs changes between pre and post NAC}

Hida, et al. reported 26 pair-matched TNBC samples TIL scores of pre-treatment biopsy and post-treatment surgical samples compared and classified as decrease, increase and no change TIL score. Patients with decreased TIL tended to have a higher rate of relapse, but the difference was not significant in that small setting. However, difference between these three groups was not significant because the absence of events in the high-TIL group [3].

In our study we report 103 pair-matched TNBC samples. Changes in TIL scores were classified as decrease, increase and no-change. The proportions of cases in each subgroup are summarized: 50 patients (48.6\%) had no-change, 26 patients $(25.2 \%)$ decrease TILs score after NAC and 27 patients (26.2\%) increase TIL score.

6 year-recurrence was $56.6 \%$ in non-change TIL group, $38.9 \%$ in decrease TIL group and $16.5 \%$ in increase TIL group $(p=0.001)$. Figure 4 illustrates disease-free survival curves according to no changes, decrease and increase TILs groups.

TILs score changes proved to have significant prognostic value ( $p=0.001$ ) regarding DFS. Median DFS in non-changesgroup was 40 months ( $95 \% \mathrm{Cl}$ 8.1-71 months), decreased TILs group 154 months (95\% Cl 9-371 months), and increased TILs group was not reached $(p=0.001)$.

Moreover, univariate and multivariate analysis confirmed TIL score changes to be an independent prognostic marker of DFS. By univariate analysis, increased TIL score between and after NAC vs. decreased or non-changes levels HR 4.01, (95\% Cl 1.69-9.59; $p=0.002)$. And by multivariate analysis TILs levels in residual disease HR $2.92(95 \% \mathrm{Cl}$ 1.05-9.66; $p=0.040$ ).

\section{Prognostic value of TILs in surgical specimens in adjuvant and NAC studies}

TILs have been evaluated in the past 10 years in nearly 16.000 patients in prospective studies with clinical follow-up data available, which highlights a rapid accumulation of evidence [3,6,9]. In adjuvant setting, BIG-02-98 trial, with 2009 breast cancer patients and 256 TNBC, suggested that higher TIL levels were associated to higher DFS and OS [6]. After that, two different adjuvant studies ECOG 2197 y 1199 with 481 TNBC patients confirmed it [18]. Therefore, Simon, et al. the value reported that prognostic value of TILs in TNBC could be considered such as Level I evidence [19].

At neoadjuvant treatment, TILs in specimen have been also evaluated. Dieci, et al. reported the prognostic value of TILs in residual disease after NAC in TNBC [20]. However, in Hida's study the difference between these three groups was not significant because the absence of events in the high-TIL 
Citation: Herrero-Vicent C, Calatrava A, Ramírez M, et al. (2019) Prognostic Value of Tumour-Infiltrating Lymphocytes Changes between Pre and Post-Neoadjuvant Treatment and in Residual Disease in Triple Negative Breast Cancer. Annals Gynecol Obstet 3(1):50-58

group [1].

In our study, at a median follow-up of 78 months, LPBC was associated with better DFS; 6-year Kaplan-Meier estimates $23 \%$ recurrence and $66.2 \%$ for patients with $L P B C$ and non-LPBC, respectively $(p=0.001)$. By univariate analysis, TILs levels in residual disease after NAC presented HR 4.27, $(95 \%$ $\mathrm{Cl}$ 2.29-7.98; $\mathrm{p}=0.001$ ). However, by multivariate analysis TILs levels in residual disease HR $1.59(95 \% \mathrm{Cl} 0.70-3.59 ; \mathrm{p}=$ 0.265).

\section{TIL's subtypes as biomarker of residual disease}

In TNBC residual disease following NAC is considered a very bad prognostic marker [21]. However, recent data has showed that the presence of TILs after NAC in residual disease confers a good prognosis in an otherwise poor prognosis group [22]. Interestingly, TILs are dissected into their different subtypes and CD8 $+\mathrm{T}$ cells are specifically analysed, a higher density of these cytotoxic cells in stroma correlates with better long-term breast cancer specific survival in the TNBC. In the case of FOXP3 Tregs, these immunosuppressive cells hinder an effective activity of cytotoxic T cells, and thus the CD8 + T cells / FOXP3 + Tregs ratio could be another interesting parameter to be evaluated in this context. Some studies have revealed that a high scoring in the CD8 + T cells/FOXP3 + Tregs ratio predict long tern relapsed-free survival and overall survival and, therefore, it could represent another valuable prognostic tool in the coming future $[15,23]$.

In addition to TILs, and their subtypes, other immune elements have been recently evaluated in breast cancer. This is the case of PDL1, one of the two ligands described for the co-inhibitory molecules PD1 which can be expressed on tumour and immune cells. The PD1/PDL1 axis is a major target for immunotherapy, and its modulation seems to be one of the main determinants for efficacy with the ne immunomodulatory monoclonal antibodies [24,25]. A recent study in 110 breast cancer patients demonstrated PDL1 positivity ( $>1 \%$ ) in $23.6 \%$ of patients, being more frequent in TNBC than in the other subtypes [3].

On the other hand, tumour-intrinsic signalling pathways that contribute to suppression of host immunity remains poorly understood. A study of residual disease in TNBC following NAC found that transcriptional activity in RAS/MAPK pathways was correlated with reduced infiltration of TILs [26]. The combination therapy with MEK inhibitors and with PD/PDL-1 produced synergistic effects in immunocompetent synergistic mouse models of TNBC [26]. This combination might be useful for promoting a more-functional immune microenvironment in patients with TNBC with residual disease after NAC that lacks TILs. Some recent trials have evaluated the efficacy of antibodies directed at PD/PDL1 in patients with TNBC with promising results, demonstrating durable responses in heavily pre-treated patients $[3,27,28]$.

TIL should be routinely used such as biomarker because is associated to a favourable balance of benefits of harms: TILs contribute clinically useful information and outweigh cost and inconvenience associated. Combination of TILS after NAC and pCR can enable patient stratification after NAC, and provides an efficient strategy for developing improved therapies.

\section{Conclusions}

In summary, prognostic impact of TIL in residual disease is confirmed in this study. Moreover, TIL score changes pre and after NAC have a prognostic value in TNBC. Although our findings may not change standard treatment options for locally advanced TNBC, TIL evaluation could be included in the standard histopathological practise as stratification factor in future clinical studies. Moreover, strategies should be considered to improve the outcome of TNBC such as immune-check point inhibitors in patients with worse prognosis.

\section{Acknowledgements}

Pathology Department Valencian Institute of Oncology.

\section{Conflict of Interest}

This research received a specific grant from Mutual Médica.

\section{References}

1. Hida Al, Sagara Y, Yotsumoto D, et al. (2016) Prognostic and predictive impacts of tumor-infiltrating lymphocytes differ between Triple-negative and HER2-positive breast cancers treated with standard systemic therapies. Breast Cancer Res Treat 158: 1-9.

2. Degnim AC, Brahmbhatt RD, Radisky DC, et al. (2014) Immune cell quantitation in normal breast tissue lobules with and without lobulitis. Breast Cancer Res Treat 144: 539-549.

3. de la Cruz-Merino L, Palazón-Carrión N, Henao-Carrasco F, et al. (2019) New horizons in breast cancer: The promise of immunotherapy. Clin Transl Oncol 21: 117-125.

4. Salgado R, Denkert C, Demaria S, et al. (2015) The evaluation of tumor-infiltrating lymphocytes (TILS) in breast cancer: Recommendations by an International TILS Working Group 2014. Ann Oncol 26: 259-271.

5. Dieci MV, Radosevic-Robin N, Fineberg S, et al. (2018) Update on tumor-infiltrating lymphocytes (TILS) in breast cancer, including recommendations to assess TILs in residual disease after neoadjuvant therapy and in carcinoma in situ: A report of the International Immuno-Oncology Biomarker Working Group on Breast Cancer. Semin Cancer Biol 52: 16-25.

6. Loi S, Sirtaine N, Piette F, et al. (2013) Prognostic and predictive value of tumor-infiltrating lymphocytes in a phase III randomized adjuvant breast cancer trial in node-positive breast cancer comparing the addition of docetaxel to doxorubicin with doxorubicin-based chemotherapy: BIG 02-98. J Clin Oncol 31: 860-867.

7. Guarneri V, Broglio K, Kau SW, et al. (2006) Prognostic value of pathologic complete response after primary chemotherapy in relation to hormone receptor status and other factors. J Clin Oncol 24: 1037-1044.

8. Balko JM, Giltnane JM, Wang K, et al. (2014) Molecular profiling of the residual disease of triple-negative breast cancers after neoadjuvant chemotherapy identifies actionable therapeutic targets. Cancer Discov 4: 232-245.

9. Savas P, Salgado R, Denkert C, et al. (2016) Clinical relevance of 
Citation: Herrero-Vicent C, Calatrava A, Ramírez M, et al. (2019) Prognostic Value of Tumour-Infiltrating Lymphocytes Changes between Pre and Post-Neoadjuvant Treatment and in Residual Disease in Triple Negative Breast Cancer. Annals Gynecol Obstet 3(1):50-58

host immunity in breast cancer: From TILs to the clinic. Nat Rev Clin Oncol 13: 228-241.

10. Brown M, Wittwer C (2000) Flow cytometry: Principles and clinical applications in hematology. Clin Chem 46: 1221-1229.

11. Denkert C, von Minckwitz G, Darb-Esfahani S, et al. (2018) Tumour-infiltrating lymphocytes and prognosis in different subtypes of breast cancer: A pooled analysis of 3771 patients treated with neoadjuvant therapy. Lancet Oncol 19: 40-50.

12. Ono M, Tsuda H, Shimizu C, et al. (2012) Tumor-infiltrating lymphocytes are correlated with response to neoadjuvant chemotherapy in triple-negative breast cancer. Breast Cancer Res Treat 132: 793-805.

13. Hida Al, Ohi Y (2015) Evaluation of tumor-infiltrating lymphocytes in breast cancer; proposal of a simpler method. Ann Oncol 26: 2351.

14. Denkert C (2013) Diagnostic and therapeutic implications of tumor-infiltrating lymphocytes in breast cancer. J Clin Oncol 31: 836-837.

15. Ali HR, Provenzano E, Dawson SJ, et al. (2014) Association between CD8+ T-cell infiltration and breast cancer survival in 12,439 patients. Ann Oncol 25: 1536-1543.

16. Demaria S, Volm MD, Shapiro RL, et al. (2001) Development of tumor-infiltrating lymphocytes in breast cancer after neoadjuvant paclitaxel chemotherapy. Clin Cancer Res 7: 3025-3030.

17. West NR, Milne K, Truong PT, et al. (2011) Tumor-infiltrating lymphocytes predict response to anthracycline-based chemotherapy in estrogen receptor-negative breast cancer. Breast Cancer Res 13: R126.

18. Denkert C, Von Minckwitz G, Brase JC, et al. (2015) Tumor-infiltrating lymphocytes and response to neoadjuvant chemotherapy with or without carboplatin in human epidermal growth factor receptor 2-positive and triple-negative primary breast cancers. J Clin Oncol 33: 983-991.

19. Simon RM, Paik S, Hayes DF (2009) Use of archived specimens in evaluation of prognostic and predictive biomarkers. J Natl Cancer Inst 101: 1446-1452.

20. Dieci MV, Criscitiello C, Goubar A, et al. (2014) Prognostic value of tumor-infiltrating lymphocytes on residual disease after primary chemotherapy for triple-negative breast cancer: A retrospective multicenter study. Ann Oncol 25: 611-618.

21. Cortazar P, Zhang L, Untch M, et al. (2014) Pathological complete response and long-term clinical benefit in breast cancer: The CTNeoBC pooled analysis. Lancet 384: 164-172.

22. Von Minckwitz G, Untch M, Blohmer JU, et al. (2012) Definition and impact of pathologic complete response on prognosis after neoadjuvant chemotherapy in various intrinsic breast cancer subtypes. J Clin Oncol 30: 1796-1804.

23. Ladoire S, Mignot G, Dabakuyo S, et al. (2011) In situ immune response after neoadjuvant chemotherapy for breast cancer predicts survival. J Pathol 224: 389-400.

24. Cimino-Mathews A, Thompson E, Taube JM, et al. (2016) PD-L1 (B7-H1) expression and the immune tumor microenvironment in primary and metastatic breast carcinomas. Hum Pathol 47: 52-63.

25. Herbst RS, Soria JC, Kowanetz M, et al. (2014) Predictive correlates of response to the anti-PD-L1 antibody MPDL3280A in cancer patients. Nature 515: 563-567.

26. Loi S, Dushyanthen S, Beavis PA, et al. (2016) RAS/MAPK activation is associated with reduced tumor-infiltrating lymphocytes in triple-negative breast cancer: Therapeutic cooperation between MEK and PD-1/PD-L1 immune checkpoint inhibitors. Clin Cancer Res 22: 1499-1509.

27. Emens LA, Braiteh FS, Cassier P, et al. (2015) Abstract 2859: Inhibition of PD-L1 by MPDL3280A leads to clinical activity in patients with metastatic triple-negative breast cancer (TNBC). Cancer Res 75.

28. Nanda R, Chow LQ, Dees EC, et al. (2016) Pembrolizumab in patients with advanced triple-negative breast cancer: Phase lb keynote-012 study. J Clin Oncol 34: 2460-2467.

DOI: $10.36959 / 468 / 466$

Copyright: (C) 2019 Herrero-Vicent C, et al. This is an open-access article distributed under the terms of the Creative Commons Attribution License, which permits unrestricted use, distribution, and reproduction in any medium, provided the original author and source are credited. 